\title{
Identity Construction: An Important Issue Among Adolescents
}

\author{
RASHMI UPRETI \\ (Research Scholar, Department of Human Development \& Family Studies, College of Home Science, P.A.U. \\ Ludhiana, India)
}

\begin{abstract}
Identity construction is another name for individuation. Individuation is the development of separate personality at a particular stage of life in which individual characteristics are possessed by a person or a person is known to others. Among adolescents identity is of great importance because it is the first time when physical development, cognitive skills and social expectations coincide that enable adolescents to understand childhood identifications in order to construct a viable pathway toward their adulthood. Personal identity develops with the changes in the adolescent brain. Identity in simple term is one's sense of self. Building of complete identity involves clarification of one's morals, ethics, and standards, as well as a commitment to a future occupation. But sometimes, adolescents pass through a stage of disorientation and role confusion and are known to be in identity crises. For the better identity construction try to engage adolescents in warm and open communication; initiate discussions that promote high-level of thinking at home and also at school; provide them opportunities to talk with adults and peers; accept the adolescent for who she or he is; encourage a young person's self-expression; take the adolescent's point of view into account.
\end{abstract}

Keywords- Gender, Identity crises, Identity theories, Peers, Personality.

\section{INTRODUCTION}

Adolescence is a distinguished period in a person's life to sum up his/her past and current experience to arrive at a sense of identity, which in turn will guide such person's future plans [1]. Therefore, adolescence is the period that witnesses the sense of identity. Adolescence is the first time in life when a person intensely contemplates the question, "Who am I?" Changes in the adolescent brain give them the tools to start building a personal identity. Identity is one's sense of self. Identity construction is also known as individuation. It is the development of separate personality at a particular stage of life in which individual characteristics are possessed by a person or a person is known to others. In psychology, identity is the conception, qualities, beliefs, and expressions that make a person (self-identity) or group (particular social category or social group) [2]. Any disturbance in the process of identity formation will lead to many consequences like time confusion, role confusion, choosing a negative identity, tendency to excel in all the situations, generalized doubt, and uncertainty [3].

\subsection{Theories on identity formation}

Many theories of development have aspects of identity formation. Two theories stand out in this regard: Erik Erikson's theory of psychosocial development (specifically the "identity versus role confusion" stage of his theory) and James Marcia's identity status theory

1.1.1 Erik Erikson's theory: Erikson belief that every person experience different crises or conflicts throughout his/her lifetime. He has divide life into eight stages and argued that every conflict of a particular stage must be resolved successfully for progression to the next of the eight stages. The particular stage by which every adolescent passes through is called "Identity versus Role Confusion." Erikson reported that this crisis can be resolved with the identity achievement, where an individual has extensively considered various goals and values, accepting some and rejecting others, and understands who they are as a unique person. If the "Identity versus Role Confusion" crisis is not solved, an adolescent probably will face confusion about future plans, particularly their roles and responsibilities in adulthood. If an adolescent fails to form one's own identity, it leads to failure to form a shared identity with others, which finally could lead to instability in many areas as an adult. 1.1.2 James Marcia's identity status theory: James Marcia (1976) explained that there are four statuses of identity; these are identity achievement, identity moratorium, identity foreclosure, and identity diffusion. These are also called as paths to identity. 
1. Identity Achievement: When an individual has resolved the identity issues by making commitments towards goals, beliefs and values after exploration of different areas, then identity is said to be achieved.

2. Identity Moratorium: This postpones identity achievement by providing temporary shelter. Moratorium refers to active exploration of other possible alternatives. This status provides opportunities for exploration. But individuals are in a state of uncertainty regarding life choices.

3. Identity Foreclosure: This occurs when adolescents accept traditional values and cultural norms, rather than determining their own values. They might also foreclose on a negative identity, the direct opposite of their parent's values or cultural norms. Foreclosure is characterized by strong commitments without much exploration of other possible alternatives.

4. Identity Diffusion (also known as Role Confusion): This is the opposite of identity achievement. In this stage, an individual has not yet resolved their identity crisis, failing to commit to any goals or values and in establishing future plans. Among adolescents, this stage is characterized by avoidance of issues and action, disorganized thinking and procrastination. Identity diffused individuals seem to drift aimlessly and are carefree.

\section{FACTORS AFFECTING ADOLESCENT IDENTITY FORMATION}

The relationships adolescents have with their peers, family, and members of their social sphere play a vital role in their development.

\subsection{Influence of Personality}

Identity status is both cause and consequence of personality characteristics. Adolescents who are conformist and obedient and who assume that absolute truth is always attainable tend to be foreclosed, whereas those who are self-indulgent and doubt they will ever feel certain about anything are more identity-diffused. Young people who are more curious and open-minded and who appreciate that they can use rational criteria to choose among alternatives are more likely to be in a state of moratorium or identity achievement [4].

\subsection{Influence of Family}

Each type of family whether biological, extended or even adoptive has their own influence on identity of adolescents. The interaction that takes place between the family members and with the individual person influences the identity. Researchers and theorists basically state that an adolescent's identity is influenced by the people around them and the environment in which they live. If a family does not have integration this will probably create identity diffusion, which means that an individual has not made commitments and does not try to make commitments. Therefore, these concepts provide evidence that a family has influence on an individual no matter if the influence be good or bad. Family also plays a critical role in adolescent identity formation by clarifying their confusion about change and transition [5].

\subsection{Influence of Culture}

Culture is learned and socially shared. Social responsibilities, sexual expression, and belief-system development vary from one culture to another. Culture affects all aspects of an individual's life. Interaction with familial, social and cultural environments helps an adolescent to develop a unique belief system. The type of attitude that a culture holds on a particular topic, can affect adolescent identity in both positive and negative way.

\subsection{Influence of Peers}

Interaction with diverse peers through school and community activities encourages adolescents to explore values and role possibilities [6]. Peer groups can have positive influences on an individual, such as academic motivation and performance; however, they can also have negative influences, such as peer pressure to engage in drug use, drinking, vandalism, stealing, or other risky behavior. Peer groups also provide opportunity to develop social skills such as empathy, leadership and sharing. Susceptibility to peer pressure increases during early adolescence, and while peers may facilitate positive social development for one another and they may also hinder it.

In support of this finding, by reviewing literature, it was concluded that achieved identity is related to better relationships with peers. Being a part of a peer group, good, positive communication with peers may provide appropriate social context for adolescent's personal identity development. Adolescents' identity development is positively related with their relationships with peers. Belonging to a peer group and good relationships with peers based on mutual respect and acceptance are positively related with adolescent identity development [7]. 


\subsection{Influence of Communication technology}

Researchers investigated changes in identity development among high school adolescents. Findings suggested that this group was significantly less committed in their identity and reported significantly higher rates of identity distress and psychological symptoms (i.e., anxiety, depression, somatization) [8]. They concluded that their findings might be due to changes in communication technology which might be affecting peer relationships and subsequently, the identity formation process.

\subsection{Influence of Social Networking Sites}

Social networking sites use provides opportunities for self-disclosure which plays a role in adolescents' identity development. Decisions about how adolescents identify themselves, the feedback they received on their decisions, and how they view their own profile in comparison to others' profiles are potential factors in individual identity.

\section{IDENTITY STATUS AND PSYCHOLOGICAL WELL-BEING}

According to identity theorists, individuals who move away from foreclosure and diffusion toward moratorium and achievement build a well-structured identity that integrates various domains. A wealth of research supports the conclusion that identity achievement and moratorium are psychologically healthy routes to a mature self-definition, whereas long-term foreclosure and diffusion are maladaptive. Although adolescents in moratorium are often anxious about the challenges they face, they resemble identity-achieved individuals in using an active, information-gathering cognitive style to make personal decisions and solve problems. They seek out relevant information, evaluate it carefully, and critically reflect on and revise their views [4].

A study investigated adolescent identity formation in relation to psychological well-being and parental attitudes (viz., acceptance, concentration, and avoidance) [9]. Analyses revealed that among boys, psychological well-being was positively correlated with identity achievement while opposite pattern emerged for diffusion. Avoidant and concentrated parental attitudes have significant positive correlates with lower identity statuses (moratorium, foreclosure, and diffusion in either gender). Also, girls in lower identity statuses experienced more avoidant and concentrated parenting.

\section{IDENTITY FORMATION AND GENDER}

Research revealed that there were statistically significant differences between adolescents with high and low Ego-identity in their overall score of Ego-identity [10]. Concerning Ideological Ego-Identity, there were statistically significant differences between males and females in both Ego-Identity achievement and EgoIdentity moratorium. They were in favor of males. Whereas, no statistically significant differences were detected between males and females in both Ego-Identity foreclosure and Ego-Identity diffusion.

In another study, gender difference in the ego identity status of higher secondary students in Cuddalore District of Tamilnadu was investigated [1]. Results indicated that the mean scores of both boys and girls were higher in identity achievement rather than other statuses such as identity moratorium, identity foreclosure and identity diffusion. It further indicated that the overall identity status of girls was higher than the boys. The boys and girls differed significantly only in the identity foreclosure and identity diffusion, they did not differ significantly in other statuses. Further, a study was conducted to investigate the pattern of identity development among adolescents of different categories [11]. From this study it was concluded that, both boys and girls were equally good at personal identity, more number of girls had high relational identity whereas high percent of boys had high social identity and collective identity too.

\section{SELF ESTEEM \& ADOLESCENT IDENTITY}

A research study on college students selected from different districts of West Bengal was conducted. The correlation analysis conducted between self-esteem and different types of identity statuses revealed that those having high identity achievement status had high self-esteem, especially in the areas of occupation and ideological belief for religion. On the other hand, adolescents who were in crisis and not made commitment had low self-esteem. The analysis also indicated that ego-identity status of students differed with respect to gender and rural-urban location [12].

\section{IDENTITY FORMATION \& PARENTAL ATTITUDE}

In identity formation, the type of relationship between the adolescents and their parents plays a significant role. For example, when there is a solid and positive relationship between parent and adolescent they are more likely to feel freedom in exploring identity options for themselves. A study found that for both boys and girls, identity formation was positively influenced by parental involvement specifically in the areas of support, social monitoring and school monitoring [13]. In opposite to this, when the relationship is not as close 
and the adolescent fears rejection from the parent, they are more likely to feel less confident in forming a separate identity from their parent(s).

\section{CONCLUSION}

Identity construction is a distinct time period in every adolescent's life. Adolescents from their own personal sense of who they are based on many things, to include the reactions and opinions of family and friends as well as the social contexts in which they live. In a summarize way we can say that "Our little efforts today could lead to a huge success tomorrow." So, for the adolescent's healthy identity development:

$>$ Engage them in warm and open communication.

$>$ Initiate discussions that promote high-level thinking at home and at school.

$>$ Provide them opportunities to participate in extracurricular activities and vocational training programs.

$>$ Provide them opportunities to talk with adults and peers who have worked through identity questions.

$>$ Accept the adolescent for who she or he is.

$>$ Respect the differences between the two of you.

$>$ Negotiate with adolescents, especially when establishing limits, and explain your reasoning.

$>$ Practice consistency in enforcing rules.

$>$ Encourage a young person's self-expression.

Take the adolescent's point of view into account when reasoning with him or her.

\section{REFERENCES}

[1] D. Janarthanam and R. Gnanadevan, Gender differences in ego-identity status of higher secondary students, International Journal of Teacher Educational Research, 3(2), 2014, 14-18.

[2] J. Paul, Despite the terrors of typologies: The importance of understanding categories of difference and identity, Interventions: International Journal of Postcolonial Studies, 17 (2), 2015, 174-195.

[3] S. Devi and Jyotsana, Identity formation: Role of social support and self esteem among Indian adolescents, The International Journal of Indian Psychology, 3(2), 2016, 4-8.

[4] M.D. Berzonsky, J. Cieciuch, B. Duriez and B. Soenens, The how and what of identity formation: Associations between identity styles and value orientations, Personality and Individual Differences, 50, 2011, 295-299.

[5] Rana and Shirali, Identity and family functioning link: An investigation of Indian youth, Journal of Psychology, 36, 2010, 266-271.

[6] B.L. Barber, M.R. Stone, J.E. Hunt and J.S. Eccles, Benefits of activity participation: The roles of identity affirmation and peer group norm sharing, in: J.L. Mahoney, R.W. Larson and J.S. Eccles (Ed.), Organized Activities as Contexts Of Development: Extracurricular Activities, After-School and Community Programs, (Mahwah: Lawrence Erlbaum Associates, 2005) 185-210.

[7] T. Rageliene, Links of adolescents identity development and relationship with peers: A Systematic literature review, Journal of Canadian Academy of Child and Adolescent Psychiatry, 25(2), 2016, 97-105.

[8] S. Berman and J. Wilson, Changing individuals in a changing world: Recent trends in identity development, Presented at the Society for Research in Identity Formation's annual convention, Philadelphia, PA, 2010.

[9] D. Sandhu, B. Singh, S. Tung and N. Kundra, Adolescent identity formation, psychological well-being, and parental attitudes, Pakistan Journal of Psychological Research, 27(1), 2012, 89-105.

[10] M.A. Diyar and A.A. Salem, Disparity of ego-identity components in relation to psychological security of adolescents, International Education Studies, 23(4), 2015, 130-135.

[11] Rani and A. Devi, Study on pattern of identity development among adolescents, International Journal of Science and Research, 5(2), 2016: 778-781.

[12] R. Basak and A. Ghosh, Ego-identity status and its relationship with self-esteem in a group of late adolescents, Journal of the Indian Academy of Applied Psychology, 34(2), 2012, 337-344.

[13] E.M. Morgan and N. Korobov, Interpersonal identity formation in conversations with close friends about dating relationships, Journal of Adolescence, 13, 2011, 32-37. 